\title{
Neutrales Rufnamengenus zwischen Grammatik und Pragmatik
}

\author{
Simone Busley und Julia Fritzinger (Mainz)
}

\begin{abstract}
In numerous German dialects and in Luxembourgish, female first names can take on both feminine and neuter gender agreement, thus leading to gender variation on a paradigmatical level and gender mismatches on a syntactical level. This is contradictory to canonical conceptions of gender systems and can be interpreted as a case of degrammaticalization. Here, grammatical gender has been refunctionalized as a socio-pragmatic marker which indicates the age and status of the woman referred to as well as the speaker's relationship to her. In some varieties, regrammaticalization of gender assignment resulted in female first names always taking neuter agreements.

The present article focusses on the reconstruction of the stages of degrammaticalization and regrammaticalization of gender assignment based on data of the research project "Das Anna und ihr Hund - Weibliche Rufnamen im Neutrum". Analyses of the data indicate that personal pronouns as the targets most prone to differing agreement played a key role in this process.
\end{abstract}

\section{$1 \quad$ Einleitung}

Im Rahmen des trinationalen Forschungsvorhabens „Das Anna und ihr Hund - Weibliche Rufnamen im Neutrum. Soziopragmatische vs. semantische Genuszuweisung in Dialekten des Deutschen und im Luxemburgischen“ (2015-2019) ${ }^{1}$ wurde das zuvor sowohl von der Dialektologie als auch der Genusforschung vernachlässigte Phänomen neutraler Frauennamen ( $\mathrm{E}$ Emma, et Maria) eingehend untersucht. Sein Verbreitungsschwerpunkt liegt im Westmitteldeutschen (Moselfränkisch, Ripuarisch) und Luxemburgischen. Nach Norden reichen die Neutra bis ins West- und Ostfälische, nach Osten über das Nordhessische bis ins Thüringische und im Süden bis ins Alemannische inkl. Schweiz und Elsass (siehe die Karte in Abschnitt 4.1). ${ }^{2}$

\footnotetext{
${ }^{1}$ Es handelt sich um ein D-A-CH-Verfahren mit Deutschland, der Schweiz und Luxemburg, gefördert durch die Deutsche Forschungsgemeinschaft (DFG), den Schweizerischen Nationalfonds (SNF) und den Fonds National de la Recherche Luxembourg (FNR), unter der Leitung von Helen Christen (Universität Freiburg i. Ü.), Peter Gilles (Universität Luxemburg) und Damaris Nübling (Johannes Gutenberg-Universität Mainz).

2 Belege aus Dialektgrammatiken und -wörterbüchern aus dem 19. Jahrhundert zeigen, dass die areale Ausdehnung historisch deutlich größer gewesen sein muss. So finden sich Erwähnungen der Neutra auch für das Ostthüringische, Ostfränkische und Schwäbische.
}

Linguistik online 107, 2/21 - http://dx.doi.org/10.13092/lo.107.7690

CC by 3.0 
Wie sich zeigt, ist die variable Genuszuweisung soziopragmatisch gesteuert, wobei Eigenschaften der Referenzperson ${ }^{3}$ (Alter, Status) sowie die Beziehung zwischen ihr und dem/der Sprecher/in ${ }^{4}$ und ggf. weiteren Gesprächsteilnehmer/innen ausschlaggebend sind (cf. Christen 1998; Nübling et al. 2013; Busley/Fritzinger 2018; Baumgartner 2019; Martin 2019): Das Neutrum indiziert Nähe und referiert primär auf junge, vertraute, verwandte Frauen und Mädchen, das Femininum dagegen markiert Distanz und gilt Respektspersonen und fremden Frauen.

Das Neutrum wird dabei nicht immer (nur) am Rufnamenartikel realisiert, sondern beispielsweise auch an Personalpronomen und Possessiva, wobei sich auch Inkongruenzen beobachten lassen, cf. das folgende Beispiel aus dem rheinfränkischen Donsieders:

(1) DONw59: ja die missten in südtirol sin

do machen de torsten un die emily ${ }^{5}$ mache werrer berchtoure

$\underline{\underline{\text { das }}}$ hat jetz a schun sei rucksack uff

,Ja, die müssten in Südtirol sein. Da machen der Torsten und die Emily wieder Bergtouren. Das hat jetzt auch schon seinen Rucksack auf.

(Fotogespräch, Donsieders, Rheinfränkisch)

Die beschriebenen Genusvarianzen widersprechen den Vorstellungen kanonischer Genussysteme, die sich u. a. dadurch auszeichnen, dass jedes Nomen ein festes, invariables Genus aufweist und das Genus eines Nomens („Controller“) an allen Genuszielen („Targets“) wie z. B. Artikel und Personalpronomen übereinstimmt (cf. Corbett/Fedden 2016: 517).

Der vorliegende Beitrag betrachtet das abweichende Genus weiblicher Rufnamen aus genusund grammatikalisierungstheoretischer Perspektive. In Abschnitt 2 nehmen wir zunächst das soziopragmatische Genus als Fall von Degrammatikalisierung in den Blick. Anschließend diskutieren wir die zentrale Rolle des Pronomens bei der Entstehung der soziopragmatisch gesteuerten Genusvariabilität (Abschnitt 3). In Abschnitt 4 gehen wir auf areale Unterschiede hinsichtlich der Neutrumfrequenz und der Genussteuerung ein und präsentieren Fallbeispiele für die verschiedenen Subsysteme. In einem abschließenden Fazit skizzieren wir hypothetische Schritte der Degrammatikalisierung von Genus zum variablen Status- und Beziehungsmarker sowie der Regrammatikalisierung des Neutrums zum weiblichen Defaultgenus (Abschnitt 5).

\section{Soziopragmatisches Genus als Fall von Degrammatikalisierung}

Nominale Klassifikationssysteme weisen unterschiedliche Grammatikalisierungsgrade auf (cf. Grinevald 2002). Vergleichsweise schwach sind sog. „Classifier“ grammatikalisiert. Hierbei handelt es sich um freie oder gebundene Morpheme mit oft noch erkennbarem lexikalischen Ursprung, cf. die Nominalklassifizierer für ,Mann` (naj> winaj, Mann') und ,Tier' (no'> nog' ,Tier') in der Maya-Sprache Jakaltek (Beispiel aus Grinevald 2002: 262):

\footnotetext{
3 Im Folgenden kurz als „R“ bezeichnet.

${ }^{4}$ Im Folgenden kurz als ,S“ bezeichnet.

5 In den Beispielen werden feminine Targets durch einfache, neutrale durch doppelte Unterstreichung hervorgehoben.
} 


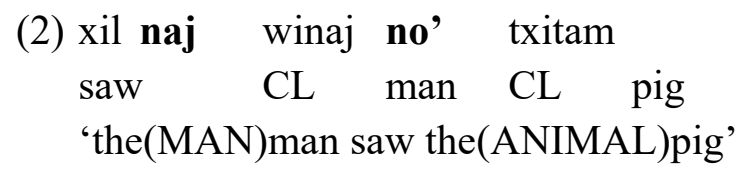

Sie klassifizieren das Nomen semantisch, indem sie Informationen über materielle, physische oder funktionale Eigenschaften des Referenzobjekts liefern. Die Zuordnung eines Classifiers zu einem Nomen kann variieren, z. B. kann ein Fluss im Burmesischen durch unterschiedliche Classifier als Picknickplatz, Angelstätte, Weg zum See etc. klassifiziert werden (cf. Corbett 1991: 136).

Genus dagegen weist einen sehr hohen Grammatikalisierungsgrad auf: Zum einen ist der Genuswert eines Nomens i. d. R. inhärent festgelegt; im Vergleich zu den beiden anderen im Deutschen vorhandenen nominalen Kategorien Numerus und Kasus, bei denen hinsichtlich des Kategorienwerts paradigmatische Wahlmöglichkeit besteht (Numerus: Singular, Plural; Kasus: Nominativ, Genitiv, Dativ, Akkusativ), handelt es sich somit bei Genus um keine echte grammatische Kategorie. Die (heutige) Funktion von Genus wird meist in der Herstellung von Kongruenzbeziehungen gesehen (cf. Corbett 1991: 320-322), im Deutschen ist es hierdurch maßgeblich an der Konstruktion von Nominalklammern beteiligt (cf. Ronneberger-Sibold 2010). Jenseits der Syntax ist die Kategorie Genus jedoch eine Form ohne echte Funktion. ${ }^{6}$ Bei Personenbezeichnungen und Rufnamen besteht zwar (zumindest im Standarddeutschen) meist Übereinstimmung zwischen Genus und Sexus (die Tante, die Anna; der Vater, der Peter), aufgrund ihrer Geschlechtsdefinitheit ist die in der Genuszuweisung enthaltene Sexusinformation jedoch i. d. R. redundant. Ausgenommen sind per se genuslose Nomen wie substantivierte Adjektive und Partizipien (der/die Angestellte) sowie Familiennamen (der/die Schmidt), bei denen das sog. „Differentialgenus“ Auskunft über das Geschlecht der Person gibt. Hier ist die Kenntnis der Referenzperson notwendig, womit Genus wortextern zugewiesen wird (cf. Busley/Fritzinger 2020). Im Folgenden beschränken wir ,semantische Kongruenz/Genuszuweisung“ im Verständnis der Corbett'schen lexemzentrierten Genuslehre auf Fälle, in denen Genus aus der Wortsemantik abgeleitet wird, und grenzen diese damit klar von der „konzeptuellen Kongruenz/Genuszuweisung“ ab (siehe Abschnitt 3.1). Letztere umfasst Fälle, in denen Genus referentiell oder soziopragmatisch zugewiesen wird.

Referentielle Genuszuweisung lässt sich insbesondere bei Eigennamen beobachten, denen Genus häufig auf Basis der Objektklasse des Namenträgers zugewiesen wird (cf. Köpcke/Zubin 2005; Fahlbusch/Nübling 2014): Je nach Objektbezug können demselben Namenkörper alle drei Genera zugewiesen werden, z. B. der Atlanta für ein Auto, die Atlanta für ein Schiff oder Motorrad, das Atlanta für ein Hotel, Restaurant oder eine Biersorte. Hier hat Nübling (2015) zufolge eine Refunktionalisierung und damit Degrammatikalisierung von Genus zur ontologischen Klassifizierung des Namenträgers stattgefunden, wodurch das referentielle Genus Merkmale von Classifiern aufweist. Dabei kann einer Objektklasse nur ein festes Genus zugewiesen werden, wodurch ein Genuswechsel immer einen Objektwechsel anzeigt. Anders verhält es sich

\footnotetext{
${ }^{6}$ Diachron können sich die weniger grammatikalisierten Classifier zu Genera entwickeln, wobei auch Zwischenstufen existieren (cf. Corbett/Fedden 2016).
} 
beim ebenfalls degrammatikalisierten soziopragmatischen Genus, das ein und dasselbe Referenzobjekt (eine weibliche Person) unterschiedlichen Beziehungskonzepten zuordnet. Genus wurde hier zur Beziehungsanzeige refunktionalisiert.

Der Definition von Norde zufolge geht Degrammatikalisierung mit einem Autonomie- oder Substanzzuwachs auf mehreren Sprachebenen einher: „Degrammaticalization is a composite change whereby a gram in a specific context gains on autonomy or substance on more than one linguistic level (semantics, morphology, syntax, or phonology).“ (Norde 2009: 119). Bei den Femineutra sind hiervon die semantische bzw. die (von Norde unerwähnte) pragmatische sowie die syntaktische Ebene betroffen. Während kanonisches Genus lediglich auf Lexemebene klassifiziert und somit keine (zusätzlichen) Informationen über das bezeichnete Objekt liefert, indizieren Femininum und Neutrum in den Dialekten mit soziopragmatischer Genuszuweisung jeweils bestimmte Eigenschaften von R (Alter, Status) und/oder die soziale Beziehung zwischen S und R. Sie wurden pragmatisch refunktionalisiert: Bei der Referenz auf weibliche Personen ist Genus nach kommunikativen Absichten frei wählbar und wird (wieder) zur grammatischen Vollkategorie. Gestützt wird dies durch die Integration von exklusiv der weiblichen Referenz dienenden Sonderformen wie ääs, ihns und lux. hatt ins Pronominalparadigma (zu deren Genese und Funktion siehe ausführlich Klein/Nübling 2019).

Zusätzlich kommt es auf (morpho-)syntaktischer Ebene zu einer Auflösung der Kongruenzbeziehungen: In den untersuchten Dialekten stimmt das Genus des Rufnamenartikels und der übrigen Targets (z. B. Personalpronomen, Possessiva) häufig nicht überein, womit eines der wichtigsten definitorischen Merkmale von Genus, nämlich die Kongruenz über alle Targettypen und syntaktischen Domänen hinweg, abgebaut wird. Das Pronominalgenus ist syntaktisch vom Controllergenus entkoppelt und pragmatisch zugewiesen. Besonders deutlich wird dies bei der neutralen Wiederaufnahme femininer Lexeme wie Tochter (siehe Abschnitt 3.2). Im nächsten Abschnitt gehen wir näher auf die Schlüsselrolle der Pronomen bei der Entstehung des soziopragmatischen Genus ein.

\section{Das Pronomen als Türöffner für die onymische Genusvariabilität}

Im Folgenden wird gezeigt, dass die soziopragmatische Genusvariabilität stark an wortartspezifische Eigenschaften geknüpft ist. Insbesondere Pronomen, von denen als anaphorische Elemente strenge formale Übereinstimmung mit ihrem Bezugswort erwartet werden könnte, erweisen sich hinsichtlich der Genuszuweisung als besonders autonom. Aufgrund ihrer starken Tendenz zur konzeptuellen Kongruenz ist ihre Rolle als Türöffner zur soziopragmatischen Genusvariabilität zu diskutieren.

\subsection{Wortartspezifische Genusvariabilität}

Genuskongruenz wird in der Regel so verstanden, dass ein kongruenzauslösendes Element (Controller) sein inhärentes Genusmerkmal auf ein weiteres, an sich genusloses Element (Target) überträgt (cf. Corbett 2006: 4), cf. Abbildung 1. 


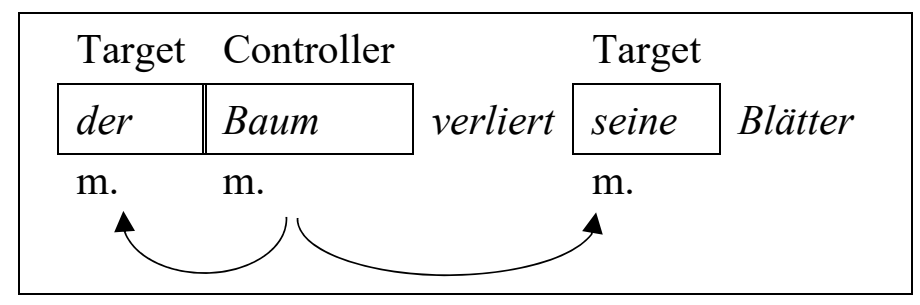

Abbildung 1: Beispiel für Genuskongruenz

Bei sog. „Hybrid nouns“ (cf. Corbett 1991: 225-260; Corbett 2006: 163-165) sind jedoch syntaktische Genusinkongruenzen beobachtbar. Dazu gehören im Deutschen geschlechtsdefinite Nomen, die dem Genus-Sexus-Prinzip widersprechen. So können bei dem klassischen Beispiel Mädchen Targets sowohl im Neutrum als auch im Femininum stehen (das Mädchen - sie/es). Corbett (2006: 163) erklärt diese Inkongruenzen mit einem nomeninhärenten Genuskonflikt: „They [= Hybrid nouns] are items which control different agreements for different targets.“ Er nimmt dabei an, dass bei hybriden Nomen formale (morphologische) und semantische Prinzipien miteinander im Konflikt stehen: Im Beispiel Mädchen wird das Neutrum vom Diminutivsuffix ausgelöst (syntaktische, grammatische oder formale Kongruenz), das Femininum durch das semantische Merkmal [+ weiblich] (semantische Kongruenz).

$\mathrm{Ob}$ ein Target eher grammatisch oder semantisch kongruiert, hängt u. a. von seiner Wortartzugehörigkeit $\mathrm{ab}$. Basierend auf sprachtypologischen Untersuchungen hierarchisiert Corbett verschiedene Targettypen auf der „Agreement Hierarchy“ hinsichtlich ihrer Affinität zur semantischen Kongruenz:

attribute $>$ predicate $>$ relative pronoun $>$ personal pronoun

(Corbett 1979: 204)

Die Wahrscheinlichkeit semantischer Kongruenz nimmt nach rechts hin zu. Für Mädchen zeigt Corbett (1991: 228, 236f.), dass attributive Elemente (Artikel, Adjektive) und Relativpronomen formal kongruierend im Neutrum stehen. Das Personalpronomen könne sowohl formal als auch semantisch kongruieren, womit Neutrum und Femininum möglich sind.

In mehreren Arbeiten wurde die Kongruenzhierarchie an das Deutsche angepasst, d. h. durch weitere Wortarten ergänzt und auch empirisch überprüft (cf. Fleischer 2012 zur Kongruenz bei ahd. wīb, nhd. Weib). Für unseren Ansatz sind solche Modifikationen der Hierarchie interessant, bei denen funktionale Eigenschaften der Wortarten als Erklärung für die Inkongruenzen herangezogen werden, siehe das Modell von Köpcke/Zubin (2009: 146) in Abbildung 2.

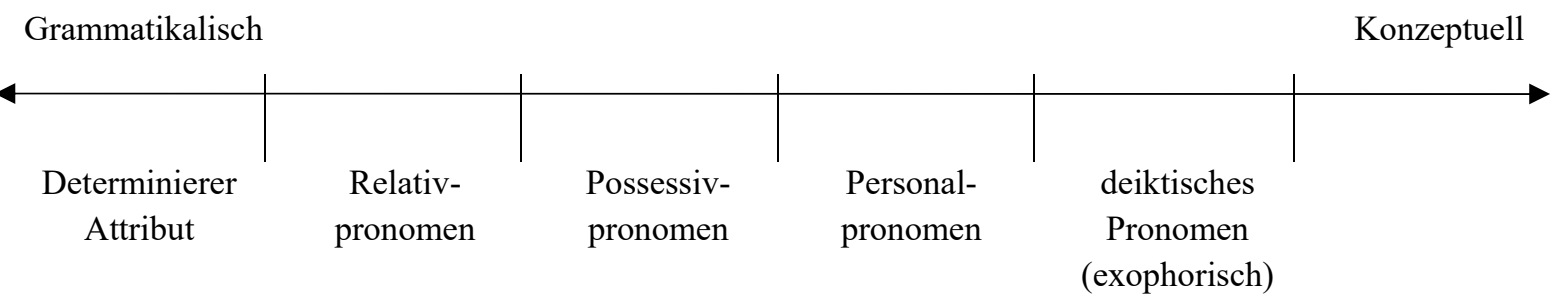

Abbildung 2: Modifikation der Agreement Hierarchy nach Köpcke/Zubin (2009: 146)

Die obige Kongruenzhierarchie ist um das Possessivpronomen erweitert, das Köpcke/Zubin zwischen Relativ- und Personalpronomen verorten. Außerdem wurde das deiktische Pronomen 
integriert. Damit zeigt sich, dass der genuslösende Konflikt anders als bei Corbett nicht im Nomen selbst angesetzt wird: Exophorische Pronomen beziehen sich exklusiv auf sprachexterne Elemente, womit ihr Genus nur referentiell gesteuert sein kann. Köpcke/Zubin (2009) (und Panther, s. u.) sprechen von „konzeptueller“ Kongruenz, da der Referent ein kognitives Konzept aufruft, das mit einem bestimmten Genus verknüpft ist (z. B. weiblich $\rightarrow$ f.). Auch das Genus anderer Targets kann konzeptuell zugewiesen werden (cf. Köpcke/Zubin 2009: 140). Je weiter man sich auf der Hierarchie nach rechts bewegt, desto wahrscheinlicher wird konzeptuelle Kongruenz.

Als Beispiel für konzeptuelle Kongruenz führen Köpcke/Zubin Weib an. Das kognitive Konzept,weiblich' sei mit dem Femininum verknüpft und werde bei Bezug auf eine weibliche Person aktiviert, womit bei Genus-Sexus-divergenten Frauenbezeichnungen Genuskonflikte ausgelöst werden können:

Fungiert Weib als Kopf einer NP, wird das Concord-Merkmal [Kongruenzmerkmal] Neutrum automatisch auf die NP-internen Targets übertragen. Wenn aber N[eutrum] als Index-Merkmal auf ein Element außerhalb der NP abgebildet werden soll, kann es zu einem Konflikt mit der vom Referenten evozierten kognitiven Eigenschaft, weiblich`samt dem damit verknüpften F[emininum] kommen.

(Köpcke/Zubin 2009: 140f.)

Panther (2009: 74) ordnet nicht Wortarten, sondern ihre Funktionen auf dieser Skala an (siehe Abbildung 3; cf. auch Köpcke et al. 2010).

\begin{tabular}{ccc} 
Spezifizieren & Modifizieren & $\begin{array}{c}\text { Verorten von } \\
\text { Referenten }\end{array}$ \\
\hline $\begin{array}{c}\text { Grammatische } \\
\text { Genuskongruenz }\end{array}$ & $\begin{array}{c}\text { Konzeptuelle } \\
\text { Genuskongruenz }\end{array}$
\end{tabular}

Abbildung 3: Pragmatische Hierarchie der Genuskongruenz nach Panther (2009: 74)

Diese pragmatische Interpretation der Corbett'schen Hierachie gründet auf der Annahme, dass konzeptuelle Genuszuweisung umso eher greift, je referentieller die Verweiskraft eines Targets ist. Artikel können Panther zufolge als spezifizierende Elemente die Identifikation des Referenzobjekts stützen, haben selbst aber keine referentielle Funktion. Damit kongruieren sie grammatisch. Modifizierer sind Attribute, auf die konzeptuelle Genuszuweisung ebenfalls nicht zugreift (*ein [n.] kleine [f.] Mädchen [n.]; cf. Panther 2009: 75).

Personalpronomen, Possessiva und Relativpronomen sind Panther zufolge Targets, die eine Verortung des Referenzobjekts vornehmen (ibd.: 76). Aus diesem Grund ist ihr Genus am stärksten konzeptuell determiniert. Panther differenziert darüber hinaus zwischen den Pronominalarten: Relativpronomen tendieren am stärksten zu grammatischer Kongruenz. Possessiva seien wiederum eine „hybride Kategorie“, da ihnen neben ihrer verortenden Funktion wie Artikeln auch eine spezifizierende Funktion zukomme. Personalpronomen als rein verortende Elemente neigen am stärksten zur konzeptuellen Genuskongruenz. Dass Pronomen sich nicht nur auf ihr Bezugsnomen, sondern auch auf den Referenten der NP beziehen können, wird häufiger angenommen (cf. z. B. Lyons 1977: 660; Kraaikamp 2017: 4). Im Folgenden wird gezeigt, dass 
sie in den untersuchten Dialekten auch auf die Beziehung zwischen Referentin und sprechender bzw. adressierter Person referieren und dabei je nach Beziehungskonzept ein anderes Genus aufweisen können. Das soziopragmatische Genus ist damit eine weitere Ausprägung der konzeptuellen Genuszuweisung.

\subsection{Konzeptuelle Genuszuweisung}

Braun/Haig (2010) identifizieren einen Alterseinfluss der Referentin bei der Genuszuweisung zu Kongruenzformen von Mädchen, der die Annahme einer konzeptuellen Genuszuweisung stützt. In ihrer empirischen Studie wurden 302 deutsche Muttersprachler/innen angeleitet, Stimulussätze unter Einbezug vorgegebener Wörter zu ergänzen (z. B. Das Mädchen war erst zwei Jahre alt, als ... [unheilbar/Leukämie/erkranken]). Die Referentinnen von Mädchen waren zwei, zwölf und 18 Jahre alt. Bei Bezug auf das 18-jährige Mädchen ist der Femininumanteil signifikant höher als bei den zwei- und zwölfjährigen Mädchen, bei denen sich die Genuszuweisung nicht signifikant voneinander unterscheidet. Braun/Haig (2010: 81) nehmen an, dass die Pubertät, ab der sich Geschlechtsmerkmale stärker ausprägen, maßgeblich für die Genuswahl ist. Hübner (in diesem Band) zeigt mit einer ähnlichen empirischen Untersuchung mit Relativpronomen, dass sexualisierende Kontexte ebenfalls das Femininum begünstigen.

Dies macht deutlich, dass Kindlichkeit bzw. sexuelle Unreife mit dem Neutrum assoziiert sind. Dabei handelt es sich aber nicht um ein von neutralen Kinderbezeichnungen unabhängiges Konzept, das auch bei Abwesenheit eines entsprechenden Bezugsnomens in die Genuszuweisung eingreift. Für deiktische Verweise setzt Köpcke (2011: 300) ungeachtet des Alters der Referentin das referentielle Genus-Sexus-Prinzip als Genuscontroller an, cf. die folgenden Beispiele (ibd.):

(3) Die sieht so aus, als ob sie sich verlaufen hätte.

(4) Die Kleine scheint sich verlaufen zu haben. (Verweis auf ein Mädchen auf der Straße.)

Das Neutrum ist laut Köpcke in diesen Fällen inakzeptabel - was im Folgenden für die Dialekte widerlegt wird. Demnach erweist sich das geschlechtskongruente (feminine oder maskuline) Pronominalgenus als von einem formalen Genusauslöser entkoppelt und zugunsten konzeptueller Klassifizierung (nach Geschlecht) von grammatischer Kongruenz entbunden.

In den von uns untersuchten Dialekten ist hier auch das neutrale Pronomen möglich und damit akzeptabel, indem es weibliche Personen nach ihrem Alter, aber auch nach dem Vertrautheitsgrad zwischen S und R klassifiziert, z. B. mit einem exophorischen Pronomen, siehe (5):

(5) kiek ens, het doe!

,Schau mal, das da!‘

(Qualitatives Interview, Kerkrade, Ripuarisch)

Diese Soziopragmatik kann auch Genusinkongruenzen bei Nomen bewirken, die im Standarddeutschen nicht genushybrid sind, z. B. bei Verwandtschaftsbezeichnungen (die Tochter - es). Hier liegen Fälle vor, die dem Standarddeutschen genau entgegengesetzt sind. Das Femininum ist bei Tochter lexikalisch determiniert, der Artikel folgt dem. An anderen Targets kann sich das konzeptuelle Neutrum durchsetzen. Der Referentin kommen Eigenschaften zu, die mit dem Neutrum verknüpft sind (jüngeres Alter, Verwandtschaft und Vertrautheit). Dies wird am genusvariablen Pronomen markiert, cf. (6): 
(6) IDm59: do hamer a noch e scheen bild lo drin von meiner schwesder [...]

$\underline{\underline{\text { dat }}}$ hat sisch ja a so versaut [...]

dat hat sisch jo mit annere sache versaut

,Da haben wir noch ein schönes Bild da drin von meiner Schwester. (...) Das hat sich ja auch so versaut. (...) Das hat sich ja mit anderen Sachen versaut. ${ }^{7}$

(Videoexperiment, Idar-Oberstein, Rheinfränkisch-Moselfränkisch)

Bei weiblichen Rufnamen ist in vielen der Dialekte auch das Artikelgenus wählbar. In diesem Fall variiert auch das Pronominalgenus. Umgekehrt gilt dies nicht: Bei genusvariablem Pronomen kann das Artikelgenus fest sein. Dies bestätigt nicht nur die Kongruenzhierarchie, sondern ist auch ein Indiz dafür, dass die Pragmatikalisierung des Neutrums in der NP-externen Domäne begonnen hat: So stellt auch Corbett (1991: 248) fest, dass Genushybridität bzw. ein Genuswechsel bei Nomen in der Regel bei den anaphorischen Pronomen beginnt.

\subsection{Vom anaphorischen Pronominalgenus zum soziopragmatischen Rufnamengenus}

Plausibel ist, dass das Neutrum ursächlich mit der häufigen Diminution von Frauenbezeichnungen (cf. Leser-Cronau 2018) und -namen (cf. Christen/Baumgartner in diesem Band; Baumgartner/Christen 2017) begründet werden kann: Sowohl Mädchen als auch diminuierte Rufnamen referierten früher hochfrequent auf weibliche Unverheiratete, die in der Gesellschaft wie in der Familie einen Kinderstatus innehatten (dazu ausführlich Busley 2019, auch Busley/Fritzinger 2018).

Der Degrammatikalisierungspfad kann dabei wie folgt skizziert werden: Das Diminutivsuffix löste das Neutrum aus, das sich auch auf NP-externe Targets übertrug. Für das Standarddeutsche kann zwar gezeigt werden, dass Genus-Sexus-divergente Nomen in der Regel sexuskongruent pronominalisiert werden, z. B. das Lenchen - sie (cf. Thurmair 2006). Dies muss jedoch nicht historisch auf die Dialekte zugetroffen haben. Dahl (2000: 110) etwa nimmt an, dass sich referentielle Kongruenz mit zunehmender Belebtheit des Referenten stärker gegenüber der grammatischen durchsetzen kann. Der historisch kindliche Status unverheirateter Frauen spricht dafür, dass sie im Vergleich zu Ehefrauen und Männern als weniger belebt konzeptualisiert wurden, sodass diminuierte Frauenbezeichnungen und -namen auch häufiger neutral pronominalisiert wurden (das Lenchen - es). Ledigen Frauen wurde die (soziale) Geschlechtsreife abgesprochen und damit das Femininum verwehrt. Durch frequente Bezugnahmen auf Ledige, die mit diminuiertem Rufnamen oder als Mädchen bezeichnet wurden, konnte sich das neutrale Pronomen mit dem Konzept, weiblich, jung, unverheiratet und niedriger Status` verknüpfen. Dafür sprechen auch neutrale Sonderpronomen wie ääs, het oder it, die exklusiv auf weibliche Personen referieren und in einigen Dialekten besonders auf Töchter und junge Mädchen verweisen (cf. Busley 2019; Klein/Nübling 2019). Dadurch wurde das pronominale Femininum in seiner Funktionsdomäne eingeschränkt und hat sich im Kontrast zum Neutrum auf die Anzeige von höherem Status und Alter verheirateter Frauen spezialisiert. ${ }^{8}$ Diese älteren, statusindizie-

\footnotetext{
${ }^{7}$ Die Äußerung unter (6) entstand im Kontext des Videoexperiments, in dem sich das Kindergartenkind Emma in einer Szene nach dem Chipsessen die Finger ableckt.

${ }^{8}$ Im Femininum referieren pronominale Vollformen wie säi, sie in vielen Dialekten speziell auf die Ehefrau.
} 
renden Funktionen femininer und neutraler Pronomina sind in älteren Dialektgrammatiken belegt, die heutige Funktion besteht primär in der Beziehungsanzeige (cf. Busley/Fritzinger 2018, Busley 2019).

Von den soziopragmatisch gesteuerten Pronominalformen ausgehend konnte sich das Neutrum anschließend auf der Kongruenzhierarchie nach links ausbreiten, wobei es bei Rufnamen nicht überall in die attributive Domäne vorgedrungen ist (siehe Abschnitt 4). Bei appellativischen Frauenbezeichnungen mit femininem Genus bleibt der Artikel normalerweise vom Neutrum verschont, allerdings gibt es auch Ausnahmen: Aus bestimmten westfälischen Dialekten etwa ist dat süster ,das Schwester' belegt (cf. Leser-Cronau 2018). In Dialekten mit hoher Neutrumfrequenz festigt sich zuerst der neutrale Artikel, während das Pronomen weiterhin, wenn auch oft nur resthaft, pragmatische Genusvariabilität aufweisen kann. Die Dialekte weisen damit unterschiedliche De- und Regrammatikalisierungsstadien von Genus auf, die Thema des nächsten Abschnitts sind.

\section{$4 \quad$ Zwischen Grammatik und Pragmatik}

\subsection{Arealität}

Die Genuszuweisung weiblicher Rufnamen in den untersuchten Dialekten weist große diatopische Unterschiede auf. Zwar setzt das Neutrum überall ein gewisses Näheverhältnis zu R voraus, jedoch variiert seine Gebrauchsdomäne zum Teil stark. Die Karte in Abbildung 4 (cf. Baumgartner et al. 2020), auf der die aktuelle Verbreitung neutraler Pronomen ${ }^{9}$ mit Referenz auf weibliche Personen nach Dialektgebiet kartiert ist, illustriert dies.

Die Daten stammen aus dem Online-Fragebogen. ${ }^{10}$ Mithilfe einer Multiple-Choice-Aufgabe wurde das Pronominalgenus bei Bezug auf die eigene Schwester erfragt, wobei feminine und neutrale Pronomen zur Wahl gestellt wurden. Der Karte liegen insgesamt 4.879 Datensätze aus ca. 1.800 Orten zugrunde. Der Anteil neutraler Pronomen variiert zwischen 2\% (Hochalemannisch) und 97\% (Luxemburgisch). Das Verbreitungsareal kann in drei Gebiete gegliedert werden, die sich durch die unterschiedliche Frequenz des Neutrums charakterisieren lassen. Am frequentesten ist es im Luxemburgischen, Moselfränkischen und Ripuarischen und in deren Übergangsgebieten (Gebiet 1). Im Rheinfränkischen und in Teilen des Niederalemannischen variiert die Frequenz des Neutrums stark in Abhängigkeit des jeweiligen Ortsdialekts (Gebiet 2). Im Schweizerdeutschen sind neutrale Pronomen vergleichsweise selten belegt (Gebiet 3). ${ }^{11}$

\footnotetext{
${ }^{9}$ Neutrale Pronomen mit weiblicher Referenz sind weiter verbreitet als der neutrale onymische Artikel, da Rufnamenartikel v. a. in den nördlicheren Dialektgebieten ungebräuchlich sind, hier weibliche Rufnamen aber dennoch neutral pronominalisiert werden können.

${ }^{10}$ Mithilfe verschiedener Aufgabentypen (Multiple-Choice-Aufgaben, Übersetzungsaufgaben, Lückentexte, Aufgaben mit freier Antwortmöglichkeit) wurde das Genus der Targets von Rufnamen und Verwandtschaftsbezeichnungen erhoben. Zudem wurden metasprachliche Fragen zum Gebrauch und der Konnotation der Neutra gestellt. Der Fragebogen wurde in sprachlich angepassten Versionen in Deutschland, Luxemburg, der Schweiz, den Niederlanden (Limburg) und im Elsass verbreitet.

${ }^{11}$ Aus dem Niederfränkischen, Ostfälischen, Thüringischen, Zentralhessischen, Ostfränkischen und Mittelalemannischen liegen vereinzelte Neutrum-Belege vor $(\leq 10$ Belege pro Gebiet).
} 


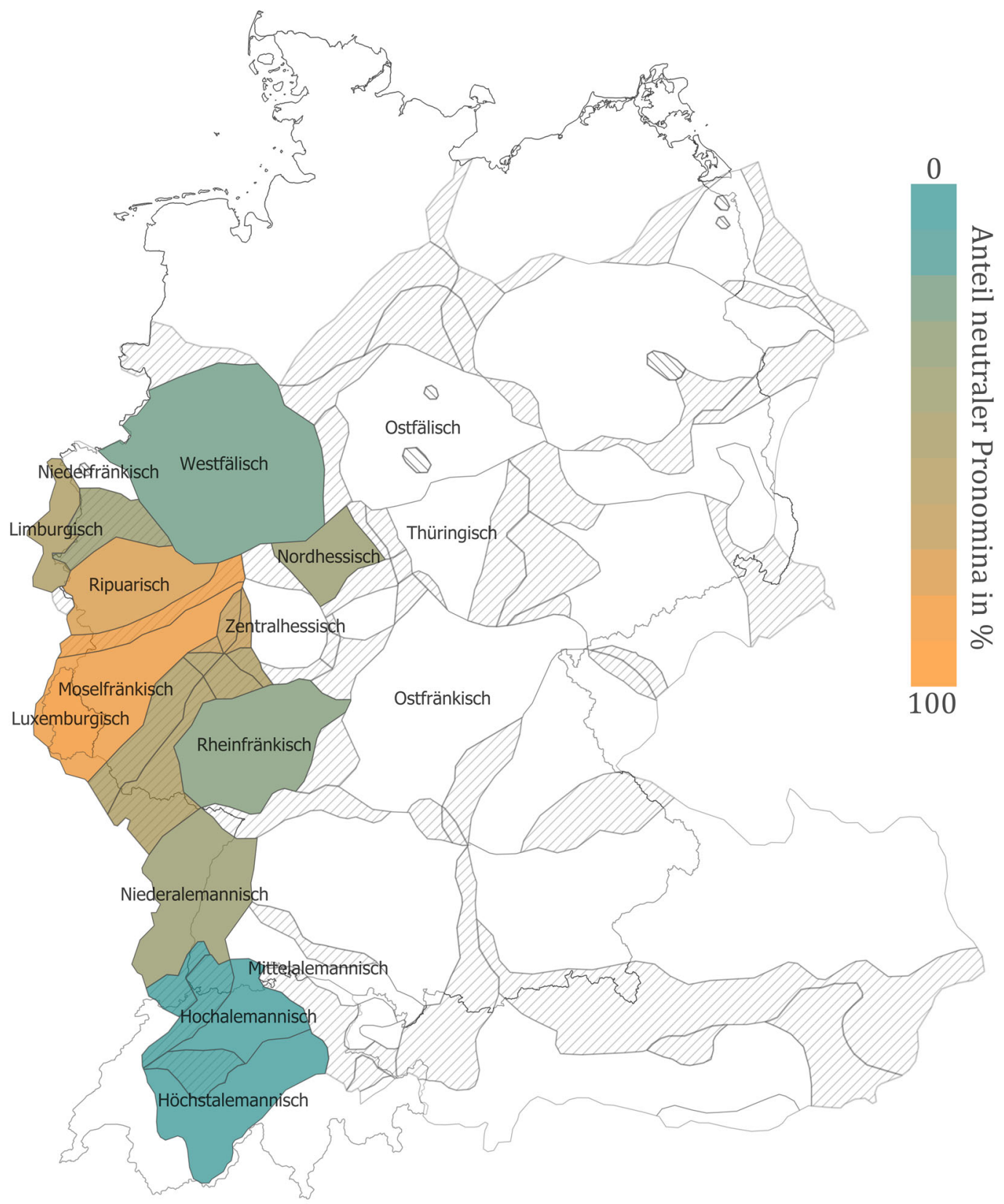

Abbildung 4: Prozentualer Anteil neutraler Pronomen nach Dialektgebiet (Online-Fragebogen, MultipleChoice-Aufgabe) nach Baumgartner et al. 2020.

Die Gebrauchsfrequenz interferiert stark mit dem Grammatikalisierungsgrad der Neutra. Dabei haben die Dialekte für die weiblichen Rufnamen zum Teil unterschiedliche Kongruenzsysteme ausgebildet, bei denen die Stabilität des Neutrums vom Target abhängen kann. Viele Dialekte weisen regelmäßig Genusinkongruenzen zwischen Rufnamenartikeln und NP-externen Targets auf. Dies veranschaulicht Tabelle 1. 


\begin{tabular}{|l|l|l|l|l|}
\hline Gebiet & Kongruenzsystem & Artikel & Pronomen & Defaultgenus \\
\hline 1 & teilvariabel & $\mathrm{N}$ & $\mathrm{N} / \mathrm{F}$ & $\mathrm{N}$ \\
\hline \multirow{3}{*}{2} & teilvariabel & $\mathrm{F}$ & $\mathrm{F} / \mathrm{N}$ & - \\
\cline { 2 - 5 } & variabel & $\mathrm{F} / \mathrm{N}$ & $\mathrm{F} / \mathrm{N}$ & - \\
\hline \multirow{3}{*}{3} & teilvariabel & $\mathrm{F}$ & $\mathrm{F} / \mathrm{N}$ & $\mathrm{F}$ \\
\cline { 2 - 5 } & variabel & $\mathrm{F} / \mathrm{N}$ & $\mathrm{F} / \mathrm{N}$ & $\mathrm{F}$ \\
\hline
\end{tabular}

Tabelle 1: Kongruenzsysteme und Defaultgenera weiblicher Rufnamen nach Gebiet

Es liegen variable und teilvariable Kongruenzsysteme vor. Konsistente Systeme, in denen die Targets weiblicher Rufnamen uneingeschränkt neutral sind (äquivalent zum Femininum im Standarddeutschen) finden sich nicht. Doch reicht das Luxemburgische durch seine hohe Neutrumfrequenz nah an ein solches System heran, nur in bestimmten Fällen können die Pronomen feminin sein. Teilvariable Systeme sind also solche, in denen der Artikel genusfest ist und das Geschlecht der Referentin anzeigt, das Pronomen aber aufgrund soziopragmatischer Steuerung variieren kann. Im Norden des Verbreitungsraums (Gebiet 1) ist der Artikel neutral, im Süden feminin (Gebiet 2 und 3). Inverse Systeme, in denen der Artikel variabel ist, Pronomen aber nicht, sind insgesamt seltener belegt. ${ }^{12}$ In Dialekten mit teilvariablem System ist meist ein Pronominalgenus der unmarkierte Default, während das andere auf spezifische Kontexte beschränkt ist. Diese Systeme weisen einen hohen (Re-)Grammatikalisierungsgrad auf, d. h. das Genus weiblicher Rufnamen festigt sich entweder zugunsten des Neutrums oder Femininums.

In variablen Systemen variieren alle Targets zwischen Femininum und Neutrum. Hierbei handelt es sich um degrammatikalisierte Systeme (cf. Fritzinger 2020). Daraus folgt, dass sowohl der Artikel als auch das Pronomen neben der Geschlechtsinformation immer auch zusätzliche soziopragmatische Informationen mitliefert. Solche Systeme sind nur in den Gebieten 2 und 3 dokumentiert. Besonders in Dialekten aus Gebiet 2 kann dabei weder Neutrum noch Femininum als Defaultgenus festgelegt werden, während Gebiet 3 zum femininen Default tendiert.

Die folgenden Abschnitte veranschaulichen die verschiedenen Systeme und ihre De- bzw. Regrammatikalisierungsgrade anhand von unterschiedlichen Fallbeispielen.

\subsection{Degrammatikalisierung}

\section{Fallbeispiel I: Mardorf (teilvariabel)}

Der Ortsdialekt des in Gebiet 2 gelegenen zentralhessischen Mardorf entspricht dem teilvariablen Typus, bei dem jedoch kein pronominales Defaultgenus identifiziert werden kann. Hier

\footnotetext{
${ }^{12}$ In einigen Schweizerdeutschen Dialekten gebrauchen v. a. jüngere Sprecher/innen den Rufnamenartikel teils noch mit variablem Genus, wohingegen die entsprechenden Pronomen konsequent feminin sind.
} 
sind sämtliche auf weibliche Rufnamen bezogenen Artikel in den erhobenen Sprachdaten invariabel feminin, wohingegen die übrigen Targets sowohl feminin als auch neutral sein können, cf. den folgenden Ausschnitt aus der Fotogespräch-Methode ${ }^{13}$ :

(7) MARw52: die ingrid ${ }^{14}$ die äh (.) die läuft marathon

immer noch

die is (-) so alt wie die martina

ochtnfuffzich

MARw34: en (.) ende fuffzich [...]

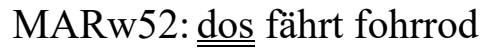

also täglichst sport

fitnessstudio fahrrad laufen $[\ldots]$

MARw34: do freut se sich

(Fotogespräch, Mardorf, Zentralhessisch)

In der schriftlichen Lückentext-Methode ${ }^{15}$ sind insgesamt 77\% (57) der auf nicht-diminuierte weibliche Rufnamen bezogenen Personalpronomen neutral. Dabei wird Genus maßgeblich vom Alter der Referentin beeinflusst, wie Auswertungen des Targetgenus nach dem absoluten Alter von $\mathrm{R}$ und dem Alter von $\mathrm{R}$ in Relation zu S zeigen, siehe Abbildung 5 und $6 .{ }^{16}$ In Bezug auf Mädchen und Frauen bis 40 Jahre sind ausnahmslos alle Personalpronomen neutral, in der Gruppe der 40- bis 60-jährigen Frauen sinkt der Neutrumanteil auf 70,6\%. Auf die älteste Referentengruppe (über 60 Jahre) wird dagegen nur mit femininen Pronomen Bezug genommen. Was das Alter von R in Relation zu S angeht, so wird auf Jüngere fast ausschließlich neutral referiert (92,2\%). Bei gleichaltrigen und älteren Referentinnen ist der Neutrumanteil mit 41,7\% (gleich) bzw. 45,5\% (älter) deutlich geringer. Das Neutrum gilt damit vor allem im Vergleich zu S jüngeren R, wobei es eine absolute Altersgrenze für das neutrale Genus zu geben scheint. Das pronominale Genus reflektiert damit das ursprüngliche, mit Kindlichkeit verknüpfte Neutrumkonzept (siehe Abschnitt 3.2).

\footnotetext{
${ }^{13}$ Bei dieser Erhebungsmethode unterhielten sich die Gewährspersonen in Kleingruppen von zwei bis drei Personen miteinander, wobei mitgebrachte Fotos aus dem privaten Fotoalbum als Gesprächsstimulus dienten.

${ }^{14}$ Die Namen realer Personen wurden zum Zweck ihrer Anonymisierung geändert.

${ }^{15}$ Den Gewährspersonen wurden dialektalisierte Lückentexte vorgelegt, in denen Targets (Artikel, Personalpronomen, Possessivartikel) zu unterschiedlichen Controllern (v. a. Rufnamen und Verwandtschaftsbezeichnungen) ergänzt werden sollten.

${ }^{16}$ Die Kontexte der Lückentexte boten Aufschluss über das ungefähre Alter der jeweiligen Referenzperson. In einem Satz geht es beispielsweise um ein kleines Mädchen, das seine Puppe verloren hat, in einem anderen um eine Rentnerin.
} 


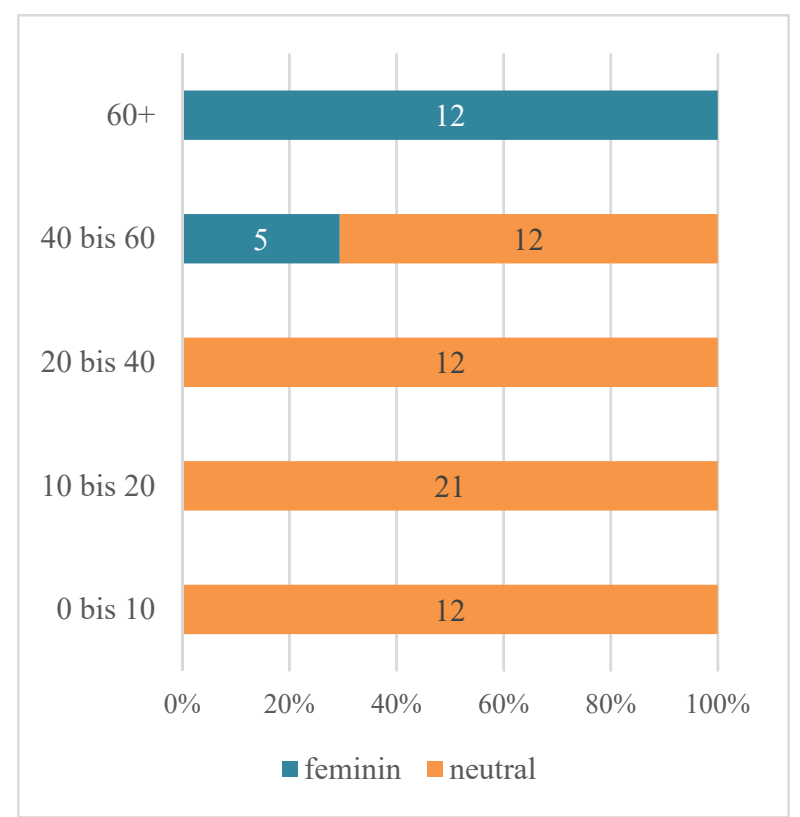

Abbildung 5: Pronominalgenus weiblicher Rufnamen nach absolutem Alter von $R$ in Mardorf (Lückentext-Methode)

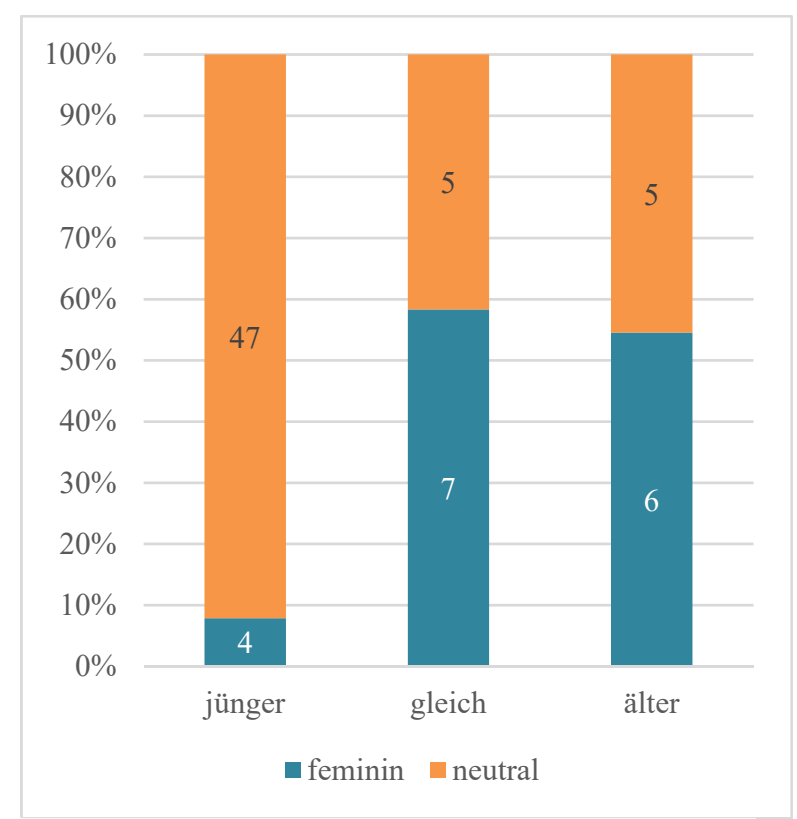

Abbildung 6: Pronominalgenus weiblicher Rufnamen nach relativem Alter von $R$ zu $S$ in Mardorf (Lückentext-Methode)

Die Steuerung nach Alter bestätigt sich auch im qualitativen Leitfadeninterview ${ }^{17}$, in dem die Gewährspersonen nach dem von ihnen gebrauchten Pronominalgenus in Bezug auf ihre Bekannten Laura (Mitte 20) und Marion (Mitte 50) gefragt werden, siehe folgender Interviewausschnitt:

(8) I: $\quad$ könnten sie über die laura sagen dos hot geburtstag?

MARw81: dos hot geburtstag

die laura hot geburtstag

das hot (-) das hot geburtstag

MARm80:das hot geburtstag ja

MARw81: ja das hot geburtstag

I: $\quad$ und bei ihrer mutter auch? also bei der marion?

MARw81: nä

do deede mer sprähe (-) däi hot geburtstog [...]

ahja also die marion is halt (--) älder

MARm80: das is e äldere person

MARw81:älder jo

da haste den underschied scho

bei de marion deet ich net spräche dos hot geburtstog

(Qualitatives Leitfadeninterview, Mardorf, Zentralhessisch)

Die Gewährsleute sind sich einig, dass ein neutrales Personalpronomen für die Mitte 20-jährige Laura verwendet werden kann, für die Mitte 50-jährige Marion jedoch das Femininum angemessener ist.

\footnotetext{
${ }^{17}$ In dieser die Erhebung abschließenden Methode wurden den Gewährsleuten konkrete Fragen zum Gebrauch der Neutra gestellt.
} 


\section{Fallbeispiel II: Donsieders (variabel)}

Ebenfalls in Gebiet 2 liegt der rheinfränkische Erhebungsort Donsieders. Wie folgende Beispiele aus dem Fotogespräch illustrieren, ist hier das Genus sowohl der Pronomen als auch der Artikel variabel, wobei auch Inkongruenzen möglich sind (siehe (11)).

(9) DONw80: die martha mim koppduch. un em luisel hat se a so e koppduch ufgezoh.

,Die Martha mit dem Kopftuch. Un dem Luischen hat sie auch so ein Kopftuch aufgesetzt.'

(10) DONw59: do misst doch s paula druf sin weil sei mann hockt jo do.

,Da müsste doch das Paula drauf sein, weil sein Mann sitzt ja da.

(11) DONw80: wollt ich mol de julia weise obs wäß wer das isch do.

,Wollte ich mal der Julia zeigen, ob es weiß, wer das ist da.

Die soziopragmatische Genussteuerung erweist sich bei näherer Betrachtung als komplexer als im zentralhessischen Mardorf. Vergleicht man zunächst die Ergebnisse zum Genus der Artikel und Personalpronomen weiblicher Rufnamen im Videoexperiment ${ }^{18}$ mit denen der Fotogespräch-Methode, wird der generelle Einfluss des Faktors ,Beziehung ' deutlich, siehe Abbildung 7 und 8: Bei der Referenz auf vertraute Personen aus dem Bekannten- und Familienkreis in den Fotogesprächen fällt der Anteil neutraler Targets deutlich höher aus (Artikel: 68,6\%, Personalpronomen: 65,6\%) als bei der Referenz auf die unbekannten weiblichen Protagonistinnen in den Videosequenzen (Artikel: 6,9\%, Personalpronomen: 28,2\%). Daneben spielt im Videoexperiment der Faktor ,Alter' eine entscheidende Rolle: Die neutralen Referenzformen betreffen hier fast ausschließlich die jüngste Protagonistin („Emma“, ca. 4 Jahre), ein kleiner Rest (3 Belege) bezieht sich mit „Miriam“ auf eine junge Frau (Mitte 20). Auf die beiden älteren Frauen („Annette“, Mitte 50; „Maria“, Mitte 70) referieren ausschließlich feminine Formen.

In den Fotogesprächen referieren die Gewährspersonen mit dem Femininum auf bekannte Personen, denen ein besonderer Status zukommt, wobei dann auch die Pronominalisierung konsequent feminin ist, cf. (9), die sich auf die Schwiegermutter der Informantin bezieht. Für vertraute gleichaltrige und ältere Frauen gelten neutrale Artikel und Pronomen, cf. (10), wo die Informantin über ihre Tante spricht. In Bezug auf jüngere weibliche Personen ergeben sich generationelle Unterschiede: Während DONw80 auf ihre Tochter mit neutralem, auf ihre (Ur-) Enkelinnen jedoch mit femininem Artikel referiert (cf. (11)), wählt DONw59 in Bezug auf Tochter und Enkelinnen ausschließlich feminine Artikel. Die entsprechenden Pronomen sind in beiden Fällen stets neutral. Es handelt sich vermutlich um einen ersten Schritt in Richtung Neutrumabbau, der hier am Artikel beginnt.

\footnotetext{
18 Die Gewährspersonen wurden gebeten, kurze Videos zu beschreiben, in denen weibliche Personen unterschiedlichen Alters mit vorgegebenen Rufnamen vorkommen.
} 


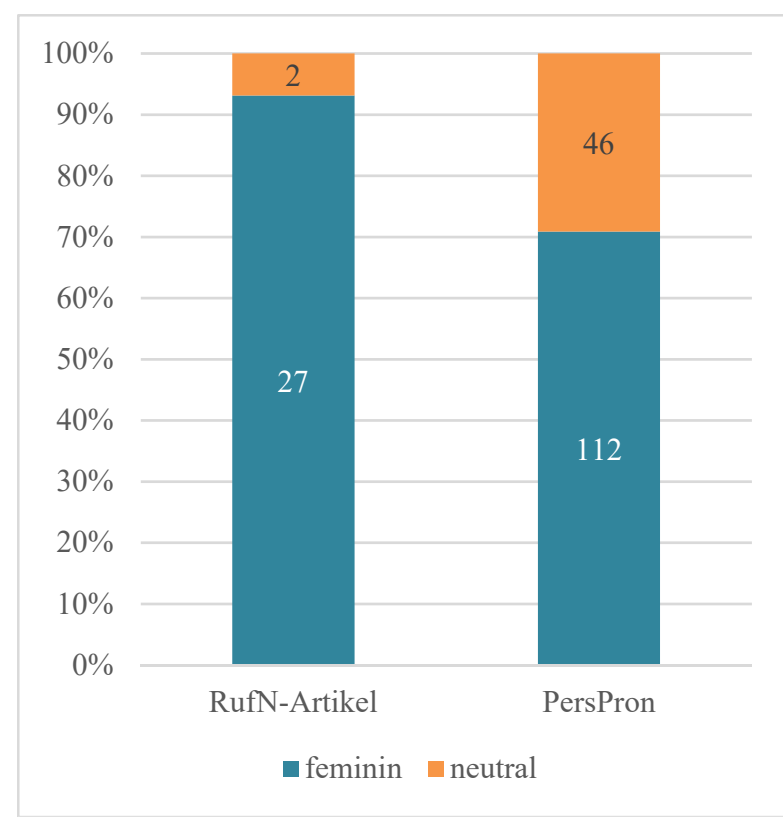

Abbildung 7: Genus der Artikel und Personalpronomen weiblicher Rufnamen in Donsieders (Videoexperiment)

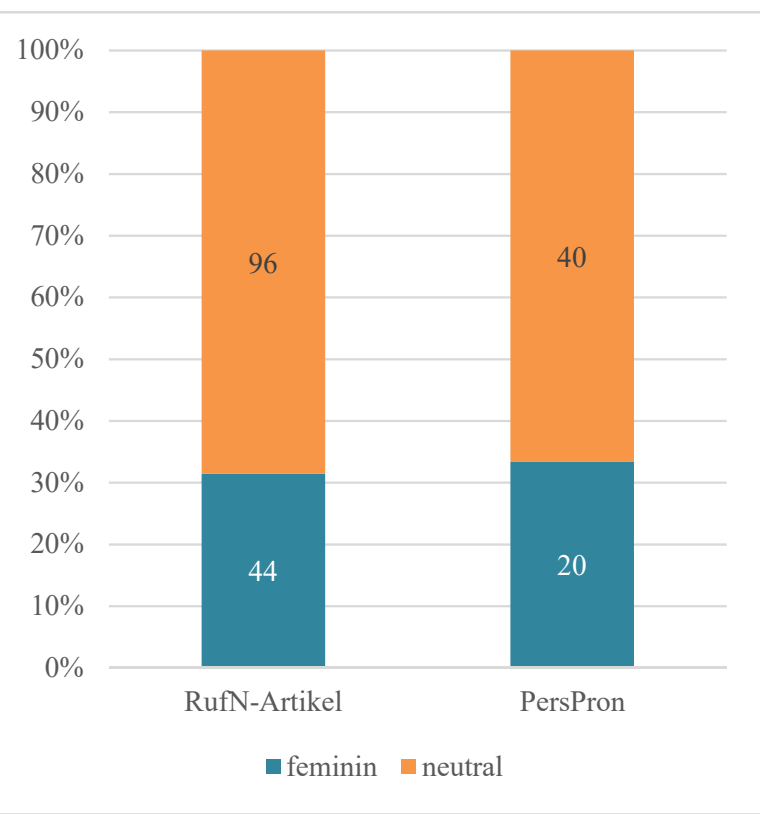

Abbildung 8: Genus der Artikel und Personalpronomen weiblicher Rufnamen in Donsieders

(Fotogespräch)

Mit davon abweichenden Genuszuweisungen gehen besondere pragmatische Effekte einher (cf. Fritzinger 2020). So kann der feminine Artikel (mit neutraler Pronominalisierung) mit Bezug auf vertraute, gleichaltrige oder ältere Frauen Distanz (Antipathie) markieren, cf. (12) aus dem Fotogespräch, in dem DONw59 auf ihre Cousine referiert:

\section{DONw59: ((stöhnt)) die brigitte}

do gugg doch mol jedes macht e normal gesicht nur das muss SO mache!

,Die Brigitte. Da schau doch mal. Jedes macht ein normales Gesicht, nur das muss SO machen!

\subsection{Regrammatikalisierung}

\section{Fallbeispiel III: Gebhardshain (teilvariabel)}

Das moselfränkisch-ripuarische Gebhardshain liegt in Gebiet 1 und entspricht dem teilvariablen Typ. Der Rufnamenartikel ist invariabel neutral. Dass das Neutrum fest an den Rufnamen gekoppelt ist, zeigt folgender Ausschnitt aus dem qualitativen Leitfadeninterview:

(13) I: $\quad$ und ähm bei den vornamen (--)

geht $d I E$ gar nicht

also die monika oder so

muss immer dann dat oder et dahin

$[\ldots]$

GEBm28: die monika det mer net sahn

GEBm56: dat geht gAr net nä

GEBm28: nä ((lacht))

$[\ldots]$ 
GEBm56: mir säächten awer hee (-)

wemmer hochdeutsch schwätten sogar dIE monika hat gesagt ja

,GEBm28: Die Monika würde man nicht sagen. GEBm56: Das geht gar nicht. GEBm28:

Nein. (...) GEBm56: Wir sagen aber hier, wenn wir Hochdeutsch sprechen sagen wir sogar

Die Monika hat gesagt, ja.

Die Ergebnisse zum Artikel und Pronominalgenus in der Lückentext-Methode in Abbildung 9 sowie im Videoexperiment in Abbildung 10 zeigen, dass das Femininum nur als Randerscheinung auftritt: In den Lückentexten sind 13,1\% der Personalpronomen feminin, in den Videobeschreibungen 11,1\% der Rufnamenartikel und 15,2\% der Personalpronomen. Das Neutrum erweist sich damit auch als pronominales Defaultgenus.

Eine genauere Betrachtung der Genuszuweisung im Videoexperiment nach Referenzperson zeigt, dass die Restdomäne des Femininums ältere Frauen betrifft: In Bezug auf die älteste Frau „Maria“ (Mitte 70) sind 37,3\% der Targets (Artikel ${ }^{19}$ und Personalpronomen) feminin, bei den jüngeren Protagonistinnen sind die Femininumanteile deutlich geringer („Emma“, ca. 4 Jahre: 1,2\%; „Miriam“, Mitte 20: 10,3\%; „Annette“, Mitte 50: 6,1\%).
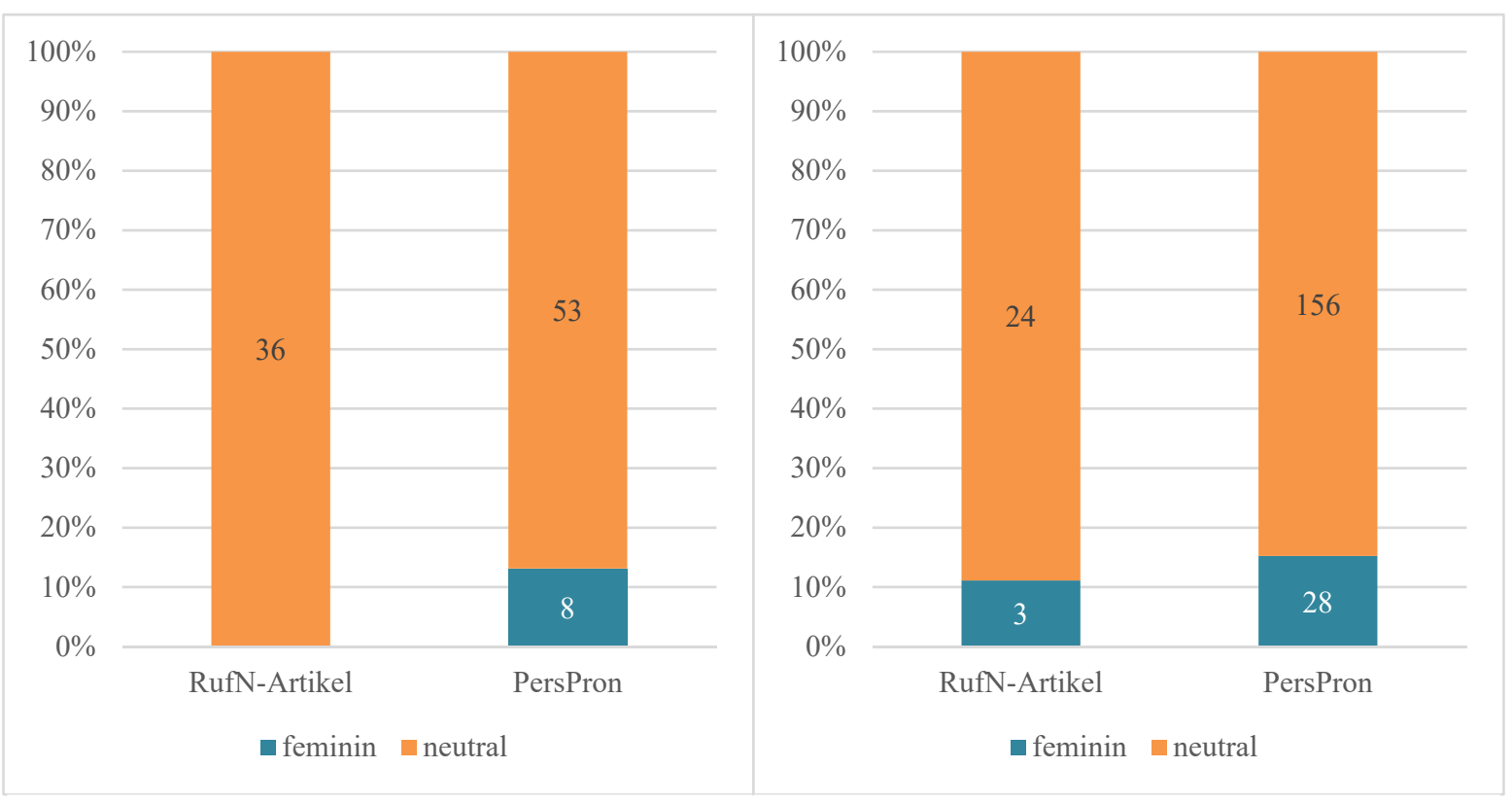

\footnotetext{
Abbildung 9: Genus der Artikel und Personalpro- Abbildung 10: Genus der Artikel und Personalpronomen weiblicher Rufnamen in Gebhardshain (Lückentext-Methode) nomen weiblicher Rufnamen in Gebhardshain (Videoexperiment)
}

\section{Fallbeispiel IV: Luxemburg (teilvariabel)}

Aufgrund seines Sonderstatus als Ausbausprache und der damit einhergehenden Standardisierung konnte sich das Neutrum im Luxemburgischen besonders stark festigen. Die Artikel weiblicher Rufnamen sind hier immer neutral und werden auch neutral pronominalisiert. In einer Multiple-Choice-Aufgabe wählten die Teilnehmer/innen zu 98,7\% das neutrale und exklusiv

\footnotetext{
19 Die mit dem (erzwungenen) Rufnamengebrauch einhergehende, aufgrund ihres Alters jedoch unpassende Nähe zu R wird hier nicht nur über das feminine Genus des Artikels, sondern auch über den Wechsel zur standardkonformen (anstatt einer dialektalen) Form korrigiert, cf. (13).
} 
weiblich referierende Pronomen hatt zur Wiederaufnahme des Rufnamens Leonie (cf. Martin 2019).

Das Neutrum entwickelt sich im Luxemburgischen zum Defaultgenus für weibliche Referenz schlechthin (cf. ibd.): In einer mithilfe der Schnëssen-App ${ }^{20}$ durchgeführten Erhebung sollten die Teilnehmer/innen zwei französische Sätze mit femininen Personalpronomen (frz. elle ,sie ${ }^{6}$ ) ins Luxemburgische übersetzen. Dabei dominieren neutrale Übersetzungen stark und nehmen in den jüngeren Generationen noch zu. Die ältesten Gewährspersonen pronominalisierten noch zu 13,3\% bzw. 14,3\% feminin, wohingegen die jüngsten fast ausschließlich Neutra wählten (zu 98,7\% bzw. 100\%). Die Generalisierung des Neutrums geht so weit, dass sogar die Namen weiblicher Tiere neutralisiert werden (cf. hierzu den Beitrag von Baumgartner et al. in diesem Band).

\section{Fallbeispiel V: Schweiz (teilvariabel)}

Eine Regrammatikalisierung kann auch in Richtung des Femininums stattfinden und in einem vollständigen Abbau des Neutrums resultieren (die Anna - sie/es $\rightarrow$ die Anna - sie), cf. die jüngere Entwicklung im Dialekt von Donsieders, auf jüngere Verwandte mit femininem Artikel (aber neutralem Pronomen) zu verweisen (siehe Abschnitt 4.2). Besonders in Varietäten der Deutschschweiz ist das Neutrum in Verbindung mit nicht-diminuierten Rufnamen heute selten. Das Femininum ist wie im Standarddeutschen das Defaultgenus bei weiblicher Referenz, das Neutrum wird pragmatisch zum Ausdruck besonderer Zuneigung oder Abwertung (cf. Baumgartner 2019) eingesetzt, die Lesart variiert nach Verwendungskontext: In Form eines „Kosegenus" ist es auf intim-familiäre Gebrauchskontexte beschränkt, außerhalb dieser privaten Sphäre wird es infolge öffentlicher Sprachkritik ${ }^{21}$ zunehmend als rückständig und sexistisch empfunden. Wie Christen/Baumgartner (in diesem Band) zeigen, bilden geschlechtsspezifisch gebrauchte Hyperkoristika auf - $i$ die heutige Kerndomäne des Neutrums: Sie referieren häufig auf Frauen und weisen dann auch regelmäßig neutrales Genus auf ( $d s$ Vreni, $d s$ Mami), wohingegen ihre männlichen Pendants nicht nur seltener, sondern i. d. R. maskulin sind ( $d r$ Hansi, $d r$ Vati). Das Neutrum hat hier eine Rückkoppelung an die Morphologie erfahren.

\section{$5 \quad$ Fazit}

Neutrale Frauennamen fordern die Genus- und Grammatikalisierungsforschung heraus. Wie wir zeigen konnten, hat eine Degrammatikalisierung und damit Refunktionalisierung der erstarrten, funktionsarmen Kategorie Genus stattgefunden, die in variabler Genuszuweisung und Auflösung von Kongruenzbeziehungen resultiert. Die neue Funktion besteht in der Beziehungsund Statusanzeige bei weiblicher Referenz.

Dieser Prozess ist von den besonders autonomen Pronomen ausgegangen. Infolge der historisch frequenten Referenz auf Mädchen und unverheiratete Frauen über diminuierte Rufnamen sowie neutrale Lexeme wie Mädchen wurden neutrale Pronomen mit dem Konzept ,weiblich, jung, unverheiratet, statusniedrig' assoziiert, das in Kontrast zum Femininum (,weiblich, verheiratet,

\footnotetext{
20 Die Spracherhebungsapp Schnëssen dient der Erforschung und Dokumentation des heutigen Luxemburgischen und ist Teil des gleichnamigen Projekts des Instituts für luxemburgische Sprach- und Literaturwissenschaft (Universität Luxemburg). Für weitere Informationen siehe Forschungsprojekt Schnëssen.

${ }^{21}$ In Deutschland wurden die Neutra bislang - wenn überhaupt - als dialektales Randphänomen reflektiert.
} 
hoher Status') trat. Im Anschluss an die syntagmatische Hybridisierung nicht-diminuierter Rufnamen (die Anna - sie/es) konnte die soziopragmatische Genussteuerung auch die attributive Domäne erobern und das Artikelgenus erfassen (die/das Anna - sie/es). Damit hat Genus den Status einer grammatischen Vollkategorie erlangt.

Durch inflationären Gebrauch des Neutrums konnte seine Soziopragmatik allmählich verblassen, was eine Regrammatikalisierung zugunsten des Neutrums ermöglichte, das sich an den Rufnamengebrauch bzw. die Geschlechtsinformation [+weiblich] koppelt. Diese geht vom Rufnamenartikel aus, wobei das weiterhin variable Pronomen noch resthaft soziopragmatisch motiviert ist (das Anna - es/sie). Die einzelnen Stufen dieses rekonstruierten diachronen Prozesses können auch synchron beobachtet werden: Fallbeispiele aus verschiedenen Varietäten haben die unterschiedlichen De- und Regrammatikalisierungsgrade (siehe Abbildung 11) illustriert. Der vorliegende Beitrag trägt damit zum tieferen Verständnis der Genese und der heutigen Ausprägungen neutraler Rufnamen bei.

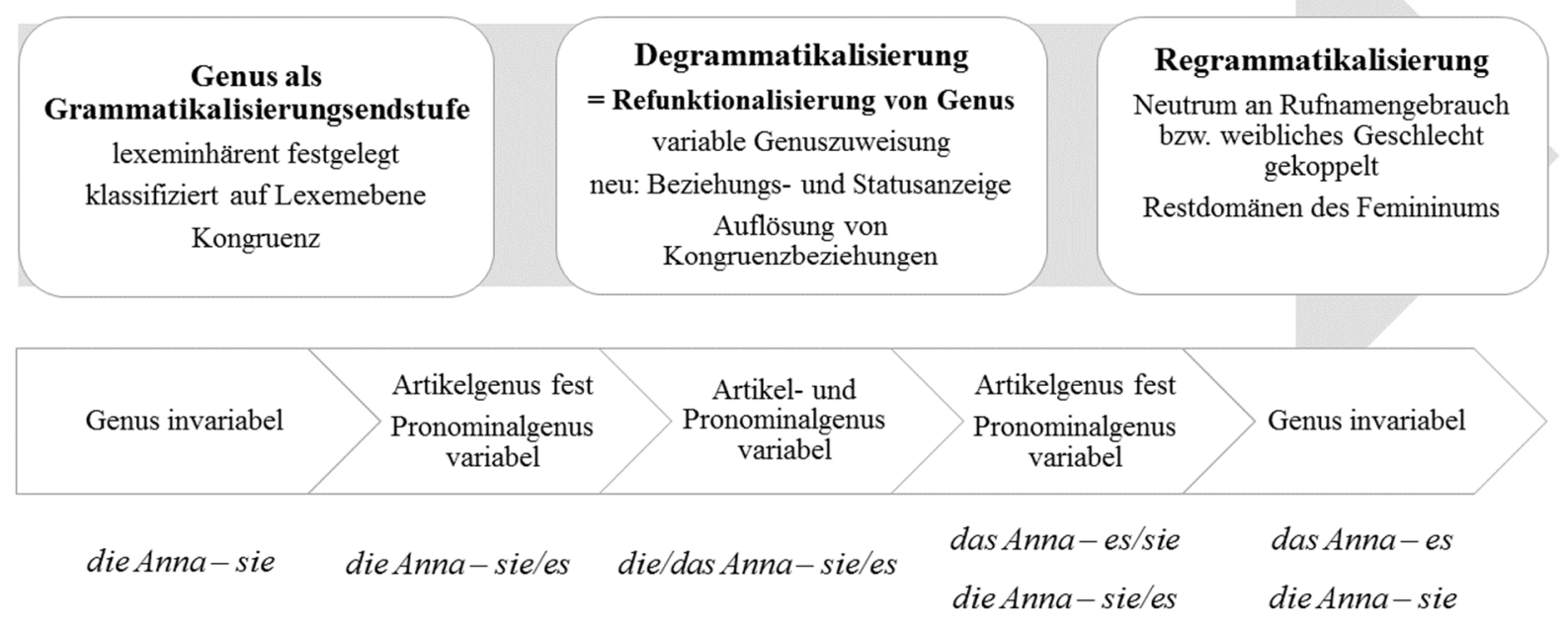

Abbildung 11: Stadien der De- und Regrammatikalisierung des weiblichen Rufnamengenus

\section{Literaturverzeichnis}

Baumgartner, Gerda (2019): „S Doris in däm wisse Fätze. Das Neutrum als affektives Genus im Namengebrauch der Deutschschweiz“. Linguistik Online 98/5: 137-156.

Baumgartner, Gerda/Christen, Helen (2017): „Dr Hansjakobli und ds Babettli - Über die Geschlechtstypik diminuierter Rufnamen in der Deutschschweiz“. Osnabrücker Beiträge zur Sprachtheorie 91: 111-145.

Baumgartner, Gerda et al. (2020): „Dat Anna, et Charlotte und s Heidi. Neutrale Genuszuweisung bei Referenz auf Frauen als überregio- nales Phänomen“. In: Christen, Helen et al. (eds.): Regiolekt - Der neue Dialekt? Akten des 6. Kongresses der Internationalen Gesellschaft für Dialektologie des Deutschen (IGDD). Stuttgart, Franz Steiner Verlag: 175-193. (=Zeitschrift für Dialektologie und Linguistik-Beihefte 182).

Baumgartner, Gerda et al. (in diesem Band): „Das Anna und ihr Hund. Weibliche Rufnamen im Neutrum?“. 
Braun, Friederike/Haig, Geoffrey (2010): „When are German 'girls' feminine? How the semantics of age influences the grammar of gender agreement". In: Bieswanger, Markus/Motschenbacher, Heiko/Mühleisen, Susanne (eds.): Language in its Socio-Cultural Context: New Explorations in Gendered, Global and Media Uses. Frankfurt, Peter Lang Verlag: 6984.

Busley, Simone (2019): Frauen im Neutrum. Empirische Studien zu mittel- und niederdeutschen Dialekten. Mainz: Unveröffentlichte Dissertation.

Busley, Simone/Fritzinger, Julia (2018): „Em Stefanie sei Mann - Frauen im Neutrum“. In: Nübling, Damaris/Hirschauer, Stefan (eds.): Namen und Geschlechter-Studien zum onymischen Un/doing Gender. Berlin/Boston, De Gruyter: 191-212. (=Linguistik - Impulse \& Tendenzen 76).

Busley, Simone/Fritzinger, Julia (2020): „De Lena sein Traum. Soziopragmatisch motivierte Genusvariabilität weiblicher Rufnamen. In: Kempf, Luise/Schmuck, Mirjam/Nübling, Damaris (eds.): Linguistik der Eigennamen. Berlin/Boston, De Gruyter: 347-376. (= Linguistik - Impulse und Tendenzen 88).

Christen, Helen (1998): „Die Mutti oder das Mutti, die Rita oder das Rita? Über Besonderheiten der Genuszuweisung bei Personen- und Verwandtschaftsnamen in schweizerdeutschen Dialekten“. In: Schnyder, André/Geith, Karl-Ernst (eds.): ,, Ist mir getroumet mîn leben “? Vom Träumen und vom Anderssein. Festschrift für Karl-Ernst Geith. Göppingen, Kümmerle: 267-281.

Christen, Helen/Baumgartner, Gerda (in diesem Band): „Annali, Ruedi, Mami. Das Diminutiv und seine Rolle bei der Enstehung und Verfestigung onymischer Neutra".

Corbett, Greville G. (1979): “The agreement hierarchy“. Journal of Linguistics 15: 203-224.

Corbett, Greville G. (1991): Gender. Cambridge: Cambridge University Press.

Corbett, Greville G. (2006): Agreement. Cambridge: Cambridge University Press.

Corbett, Greville G./Fedden, Sebastian (2016): „Canonical Gender“. Journal of Linguistics 52/3: 495-531.

Dahl, Östen (2000): „Animacy and the notion of gender“. In: Unterbeck, Barbara (ed.): Gender in Grammar and Cognition. I: Approaches to Gender. Berlin/New York, De Gruyter: 99115.

Fahlbusch, Fabian/Nübling, Damaris (2014): „Der Schauinsland - die Mobiliar - das Turm. Das referentielle Genus bei Eigennamen und seine Genese". Beiträge zur Namenforschung 49/3: 245-288.

Fleischer, Jürg (2012): „Grammatische und semantische Kongruenz in der Geschichte des Deutschen: Eine diachrone Studie zu den Kongruenzformen von ahd. wìb, nhd. Weib“. Beiträge zur Geschichte der deutschen Sprache und Literatur (PBB) 134/2: 163-203.

Forschungsprojekt Schnëssen: infolux.uni.lu/schnessen [12.08.2020].

Fritzinger, Julia (2020): Genus pragmatikalisiert. Eine empirische Untersuchung zu neutralen Referenzen auf Frauen im Rheinfränkischen und Niederalemannischen. Mainz: Unveröffentlichte Dissertation.

Grinevald, Colette (2002): „Making sense of nominal classification systems. Noun classifiers and the grammaticalization variable“. In: Wischer, Ilse/Diewald, Gabriele (eds.): New reflections on grammaticalization. Amsterdam, Benjamins: 259-275. 
Hübner, Julia (in diesem Band): „Genus und Sexus im Konflikt. Kongruenzformen hybrider Nomina im Sprachproduktionsprozess“.

Klein, Andreas/Nübling, Damaris (2019): „,Was ist es mit diesem grammatisch ungeheuerlichen ,ihns“'? Zu Form und Funktion von alem. ääs, ihns und lux. hatt“. Linguistik Online 98/5: 51-76.

Köpcke, Klaus-Michael (2011): „Grammatikalität und Akzeptabilität. Zwei für den Grammatikunterricht zentrale Begriffe verstehen lernen“. In: Köpcke, Klaus-Michael/Ziegler, Arne (eds.): Grammatik - Lehren, Lernen, Verstehen. Zugänge zur Grammatik des Gegenwartsdeutschen. Berlin, De Gruyter: 287-304. (= Germanistische Linguistik 293).

Köpcke, Klaus-Michael/Panther, Klaus-Uwe/Zubin, David (2010): „Motivating grammatical and conceptual gender agreement in German“. In: Schmid, Hans-Jörg/Handl, Susanne (eds.): Cognitive foundations of linguistic usage patterns. Berlin/New York, De Gruyter: 171-194.

Köpcke, Klaus-Michael/Zubin, David (2005): „Nominalphrasen ohne lexikalischen Kopf - Zur Bedeutung des Genus für die Organisation des mentalen Lexikons am Beispiel der Autobezeichnungen im Deutschen“. Zeitschrift für Sprachwissenschaft 24: 93-122.

Köpcke, Klaus-Michael/Zubin, David (2009): „Genus“. In: Hentschel, Elke/Vogel, Petra M. (eds.): Deutsche Morphologie. Berlin, De Gruyter: 132-154.

Kraaikamp, Margot (2017): Semantic versus lexical gender. Synchronic and diachronic variation in Germanic gender agreement. Utrecht: LOT.

Leser-Cronau, Stephanie (2018): Kongruenz bei Genus-Sexus-Divergenz in den deutschen Dialekten. Untersuchungen zu Lexical Hybrids, Rufnamen und Verwandtschaftsbezeichnungen. Marburg: Dissertation.

Lyons, John (1977): Semantics. Band 2. Cambridge etc.: Cambridge University Press.

Martin, Sara (2019): „Hatt or si? Neuter and feminine gender assignment in reference to female persons in Luxembourgish“. Language Typology and Universals 72/4: 573-602.

Norde, Muriel (2009): Degrammaticalization. Oxford: Oxford University Press.

Nübling, Damaris (2015): „Die Bismarck - der Arena - das Adler. Vom Drei-Genus- zum Sechs-Klassen-System bei Eigennamen im Deutschen: Degrammatikalisierung und Exaptation“. Zeitschrift für Germanistische Linguistik 43/2: 307-344.

Nübling, Damaris/Busley, Simone/Drenda, Juliane (2013): „Dat Anna und s Eva - Neutrale Frauenrufnamen in deutschen Dialekten und im Luxemburgischen zwischen pragmatischer und semantischer Genuszuweisung“. Zeitschrift für Dialektologie und Linguistik 80/2: 152196.

Panther, Klaus-Uwe (2009): „Grammatische versus konzeptuelle Kongruenz. Oder: Wann siegt das natürliche Geschlecht?“" In: Brdar-Szabó, Rita et al. (eds.): An der Grenze zwischen Grammatik und Pragmatik. Frankfurt, Peter Lang Verlag: 67-86.

Ronneberger-Sibold, Elke (2010): „Die deutsche Nominalklammer: Geschichte, Funktion, typologische Bewertung“. In: Ziegler, Arne (ed.): Historische Textgrammatik und historische Syntax des Deutschen. Traditionen, Innovationen, Perspektiven. Berlin/New York, De Gruyter: $1-36$.

Thurmair, Maria (2006): „Das Model und ihr Prinz. Kongruenz und Texteinbettung bei GenusSexus-Divergenz“. In: Deutsche Sprache 34, 191-220. 